\title{
VISION: a video and image dataset for source identification
}

\author{
Dasara Shullani ${ }^{1}$, Marco Fontani ${ }^{1,2}$, Massimo luliani ${ }^{1,2}$, Omar Al Shaya ${ }^{1,3}$ and Alessandro Piva $a^{1,2^{*}}$ (D)
}

\begin{abstract}
Forensic research community keeps proposing new techniques to analyze digital images and videos. However, the performance of proposed tools are usually tested on data that are far from reality in terms of resolution, source device, and processing history. Remarkably, in the latest years, portable devices became the preferred means to capture images and videos, and contents are commonly shared through social media platforms (SMPs, for example, Facebook, YouTube, etc.). These facts pose new challenges to the forensic community: for example, most modern cameras feature digital stabilization, that is proved to severely hinder the performance of video source identification technologies; moreover, the strong re-compression enforced by SMPs during upload threatens the reliability of multimedia forensic tools. On the other hand, portable devices capture both images and videos with the same sensor, opening new forensic opportunities. The goal of this paper is to propose the VISION dataset as a contribution to the development of multimedia forensics. The VISION dataset is currently composed by 34,427 images and 1914 videos, both in the native format and in their social version (Facebook, YouTube, and WhatsApp are considered), from 35 portable devices of 11 major brands. VISION can be exploited as benchmark for the exhaustive evaluation of several image and video forensic tools.
\end{abstract}

Keywords: Dataset multimedia forensics, Image forensics, Video forensics, Source identification

\section{Introduction}

In the last decades, visual data gained a key role in providing information. Images and videos are used to convey persuasive messages to be used under several different environments, from propaganda to child pornography. The wild world of web also allows users to easily share visual contents through social media platforms. Statistics [1] show that a relevant portion of the world's population owns a digital camera and can capture pictures. Furthermore, one third of the people can go online and upload their pictures on websites and social networks. Given their digital nature, these data also convey several information related to their life cycle (e.g., source device, processing they have been subjected to). Such information may become relevant when visual data are involved in a crime. In this scenario, multimedia forensics (MF) has

\footnotetext{
*Correspondence: alessandro.piva@unifi.it

'Department of Information Engineering, University of Florence, Via di S. Marta, 3, 50139 Florence, Italy

${ }^{2}$ FORLAB, Multimedia Forensics laboratory, PIN Scrl, Piazza G. Ciardi, 25, 59100 Prato, Italy

Full list of author information is available at the end of the article
}

been proposed as a solution for investigating images and videos to determine information about their life cycle [2]. During the years, the research community developed several tools to analyze a digital image, focusing on issues related to the identification of the source device and the assessment of content authenticity [3].

Generally, the effectiveness of a forensic technique should be verified on image and video datasets that are freely available and shared among the community. Unfortunately, these datasets, especially for the case of videos, are outdated and non-representative of real case scenarios. Indeed, most multimedia contents are currently acquired by portable devices that keep updating year by year. These devices are also capable to acquire both videos and images exploiting the same sensor, thus opening new investigation opportunities in linking different kind of contents [4]. This motivates the need for a new dataset containing a heterogeneous and sufficiently large set of visual data-both images and videos-as benchmark to test and compare forensic tools.

In this paper, we present a new dataset of native images and videos captured with 35 modern smartphones/tablets 
belonging to 11 different brands: Apple, Asus, Huawei, Lenovo, LG electronics, Microsoft, OnePlus, Samsung, Sony, Wiko, and Xiaomi.

Overall, we collected 11,732 native images; 7565 of them were shared through Facebook, in both high and low quality, and through WhatsApp, resulting in a total of 34,427 images. Furthermore, we acquired 648 native videos, 622 of which were shared through YouTube at the maximum available resolution, and 644 through WhatsApp, resulting in a total of 1914 videos $^{1}$.

To exemplify the usefulness of the VISION dataset, we test the performance of a well-known forensic tool, i.e., the detection of the sensor pattern noise (SPN) left by the acquisition device [5] for the source identification of native/social media contents; moreover, we describe some new opportunities deriving by the availability of images and videos captured with the same sensor to find a solution to current limits present in the literature. In particular, the proposed dataset contains several devices featuring in-camera digital stabilization, that is known to threaten source identification based on sensor pattern noise. Indeed, in most papers related to SPN [6-9], digitally stabilized videos are ignored, either by turning the stabilization off or considering non-stabilized devices only. This is unrealistic, considering that most common modern devices (e.g., Apple iPhones) are equipped with an in-camera digital stabilization system that cannot be turned off without resorting to third party applications.

The remaining part of the paper is organized as follows: in Section 2, we review the currently available datasets for image and video forensics and their current limitations; in Section 3, a complete description of the VISION dataset is provided for both native and social media contents; in Section 4, the dataset is exploited to evaluate some wellknown forensic applications and to taste new research opportunities. Section 5 draws concluding remarks.

Eventually, we include in the Appendix additional information that can be useful to perform a deeper analysis on the available visual contents.

\section{Motivation}

In the field of digital image and video forensics, only few datasets have been made available to the research community, especially for the source device identification problem. This fact is a strong limitation for research advancement in our area.

One of the first datasets adopted in the multimedia forensic community is the UCID database [10], originally designed for the evaluation of image retrieval techniques. Such dataset includes 1338 uncompressed images stored in the TIFF format, but their size is very small, either $512 \times 384$ or $384 \times 512$ pixels.

The first sufficiently large and publicly available image database specifically designed for forensic applications is the Dresden Image Database [11, 12]. This dataset includes images of various indoor and outdoor scenes acquired from 73 devices, selected from 25 camera models spanning most important manufacturers and quality ranges. All cameras were configured to the highest available JPEG quality setting and maximum available resolution, and, when supported by the device, also lossless compressed images were stored. The image resolution ranges from $3072 \times 2304$ to $4352 \times 3264$ pixels, for a total of 16,961 JPEG images, 1491 RAW (unprocessed) images, 1491 RAW images processed in Lightroom 2.5, and 1491 RAW images processed in DCRaw 9.3. Since 2010, this dataset has been used by most of the works dealing with benchmarking of source identification methods.

More recently, RAISE (RAw ImageS datasEt) was presented [13]: it is a collection of 8156 raw images including a wide variety of both semantic contents and technical parameters. Three different devices (a Nikon D40, a Nikon D90, and a Nikon D7000) are employed, and the images are taken at very high resolution $(3008 \times 2000,4288 \times 2848$, and $4928 \times 3264$ pixels) and saved in an uncompressed format (Compress Raw 12-bit and Lossless Compress Raw 14-bit) as natively provided by the employed cameras. Each image is also assigned one of seven possible categories, namely, "outdoor," "indoor," "landscape," "nature," "people," "objects," and "buildings." In the framework of the European project REWIND, a set of 200 uncompressed images acquired with a Nikon D60 camera were also made available [14] (among other sets for splicing detection, copy-move forgeries and recapture videos, all including a few number of samples). There are also other datasets, not cited here, that have been designed more for image tampering detection than for source identification, and thus no or little information is provided about the device generating the images.

As to digital videos, in the literature, there are very few datasets designed to be used in forensic scenarios; one of them is the SULFA [15], created by the University of Surrey. It collects 150 videos, each 10-s long, at $30 \mathrm{fps}$ with a resolution of $320 \times 240$ pixels. The native videos are given compressed in H.264/AVC and MJPEG, for each camera: a Canon SX220, a Nikon S3000, and a Fujifilm S2800HD. Authors designed the dataset to be used for cloning detection, performed by means of Adobe Photoshop CS3 and Adobe After Effect CS5 [15]. The SULFA dataset was also extended by the REWIND dataset [16]; anyway, these datasets are less interesting for video source identification, since they contain few digital cameras only and no smartphone, while we know smartphones are the most representative kind of device today, especially for applications on social media platforms. Recently, the video tampering dataset (VTD) was provided by Al-Sanjary et al. [17]. The VTD, focused on video tampering detection on videos collected from the YouTube 
platform, is composed by 33 downloaded videos, 16-s long, at 30 fps with a HD resolution. The original dataset is subdivided into four subsets: one containing unaltered videos; one with videos created by splicing; one with videos manipulated by copy-move; and one with videos tampered by swapping frames. Although they use a social media platform to acquire videos and provide interesting tampering techniques, there are not useful information related to the camera or device used.

The previous review shows that all currently available datasets consider mainly images, and the ones containing videos are not significant for video source identification; moreover, it is not possible to investigate relationships between images and videos acquired with the same sensor: this fact is a strong limitation, since $85 \%$ of shared media are captured using smartphones, which use the same sensor to capture both images and videos. Finally, another limit in the state-of-the-art is represented by the lack of a collection of controlled content coming from social media platforms, like Facebook, YouTube, and WhatsApp; indeed, recent multimedia forensic applications would take advantage in having a large dataset containing such kind of contents: for instance, in [6], the authors address the performance of identifying the source of YouTube videos but limiting to a scenario with videos belonging to 8 webcams of the same model (Logitech Quickcam STX). Similarly, Bertini et al. [18] propose to extract the Sensor Pattern Noise from images to identify fake social media accounts, but the technique was tested on 5 mobile devices only, with 200 images each.

\section{The VISION Dataset}

Images and videos have been acquired from each mobile device by following a specific procedure. First of all, the captured contents refer to the best-quality camera available in the device; in general, the one positioned on the upper rear of the device. Moreover, the devices were configured, when possible, with the highest quality and resolution available (usually the default one for Apple devices but not necessarily for Android ones).

VISION is mainly thought for video and image source identification applications; as a consequence, we organized the data collected from each device into two folders, (see Fig. 1 for an example), namely:

- Images: containing native and social exchanged images. We captured images, mainly in landscape mode, representing flat surfaces (e.g., skies or walls), here defined as Flat, and generic images, here defined as Nat, for which there are no limitations on orientation or scenario, as it can be seen in Fig. 2. In addition, the Nat images were exchanged via the Facebook and WhatsApp social media platforms.

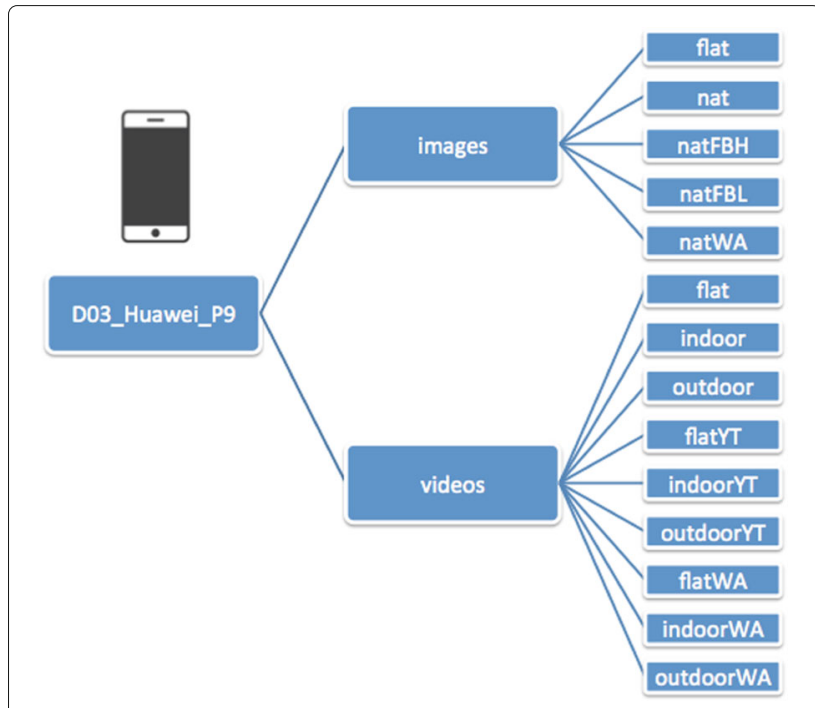

Fig. 1 VISION folder organization

- Videos: containing native and social exchanged videos, acquired mainly in landscape mode. The collected videos represent flat, indoor, and outdoor scenarios. The flat scenario includes videos belonging to flat surfaces such as walls and skies. The indoor scenario comprises videos representing offices or stores, and the outdoor scenario contains videos of open areas such as gardens. For each scenario, we used three different acquisition modes: still mode, where the user stands still while capturing the video; move mode, where the user walks while capturing the video; panrot mode, where the user performs a recoding combining a pan and a rotation. Furthermore, the videos belonging to each scenario were exchanged via YouTube and WhatsApp social media platforms.

The structure depicted in Fig. 1 is maintained also in the naming convention. The contents collected from each device are stored in its root folder named ID_Brand_Model as in D01_Samsung_GalaxyS3 Mini. Then, we distinguish between images and videos, within each of them, we have the native content folders and the social ones. A native flat image is called by convention as ID_I_flat_XXXX.jpg as in D01_I_flat_0001.jpg, where ID is the device identifier, $\bar{I}$ identifies it as an image content, flat identifies the subfolder and the type of image, while $\mathrm{XXXX} . \mathrm{jpg}$ is an incremental number. Similarly, the video content naming is ID_V_scenario_mode_XXXX.mp4 as in D01_V_flat_panrot_0001.mp4, where V identifies the video content, scenario and mode refer respectively to the area and the modality of the acquisition procedure. The so described naming 

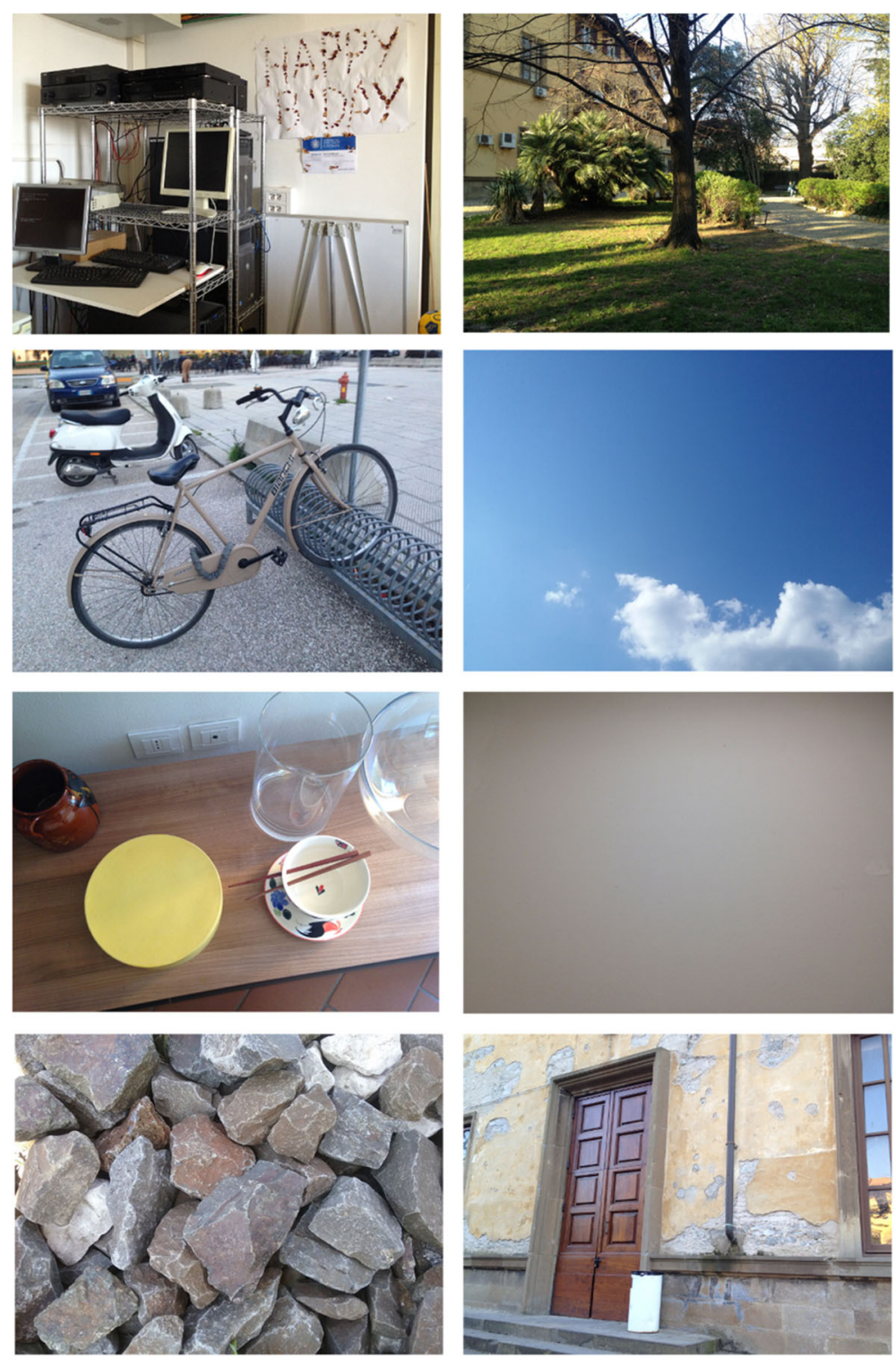

Fig. 2 Some examples of the images included in the proposed dataset

convention is also applied to the social folders represented in Fig. 1: an image uploaded to Facebook in low quality will be named D01_I_natFBL_0001.jpg, an image uploaded to Facebook in high quality will be named D01_I_natFBH_0001.jpg, while a video exchanged through WhatsApp will be named D01_V_flatWA_panrot_0001.mp4.

\subsection{Main features}

VISION is composed by 35 mobile devices from low-, middle-, and high-price range. There are 13 Apple devices, including iPhones and iPads. There are 8 Samsung devices including Galaxy phones and tablets. There are 5 Huawei and 2 OnePlus phones. Furthermore, we gathered one device for the following brands: Asus, Lenovo, LG electronics, Microsoft, Sony, Wiko, and Xiaomi. We collected a few devices of the same brand and model namely: two iPhone 4S, two iPhone 5, three iPhone 5c, two iPhone 6 , and two GalaxyS3Mini. The employed devices had installed the following operating systems: iOS from 7.x to 10.x, Android from 6.x Marshmallow to 7.x Nougat, and the Windows Phone OS 8.1 Update 2.

In Table 1, we summarize the main features of the complete dataset. For each device, we report the Brand, Model, a unique identifier $I D$, and the number of collected videos and images with their corresponding resolutions.

In Table 1, we also clarify whether videos were captured using in-camera digital stabilization: the reader can see 
Table 1 Devices main features

\begin{tabular}{|c|c|c|c|c|c|c|c|c|c|c|}
\hline Brand & Model & ID & DStab & $\mathrm{HDR}$ & VR & \#Videos & $\mathbb{I R}$ & \#lmages & \#Flat & \#Nat \\
\hline Apple & iPad 2 & D13 & Off & $F$ & $1280 \times 720$ & 16 & $960 \times 720$ & 330 & 159 & 171 \\
\hline Apple & ¡Pad mini & D20 & On & $\mathrm{F}$ & $1920 \times 1080$ & 16 & $2592 \times 1936$ & 278 & 119 & 159 \\
\hline Apple & iPhone 4 & D09 & Off & $\mathrm{T}$ & $1280 \times 720$ & 19 & $2592 \times 1936$ & 326 & 109 & 217 \\
\hline Apple & iPhone 4S & D02 & On & $\mathrm{T}$ & $1920 \times 1080$ & 13 & $3264 \times 2448$ & 307 & 103 & 204 \\
\hline Apple & iPhone 4S & D10 & On & $\mathrm{T}$ & $1920 \times 1080$ & 15 & $3264 \times 2448$ & 311 & 133 & 178 \\
\hline Apple & iPhone 5 & D29 & On & $\mathrm{T}$ & $1920 \times 1080$ & 19 & $3264 \times 2448$ & 324 & 100 & 224 \\
\hline Apple & iPhone 5 & D34 & On & $\mathrm{T}$ & $1920 \times 1080$ & 32 & $3264 \times 2448$ & 310 & 106 & 204 \\
\hline Apple & iPhone 5c & D05 & On & $\mathrm{T}$ & $1920 \times 1080$ & 19 & $3264 \times 2448$ & 463 & 113 & 350 \\
\hline Apple & iPhone $5 c$ & D14 & On & $\mathrm{T}$ & $1920 \times 1080$ & 19 & $3264 \times 2448$ & 339 & 130 & 209 \\
\hline Apple & iPhone $5 c$ & D18 & On & $\mathrm{T}$ & $1920 \times 1080$ & 13 & $3264 \times 2448$ & 305 & 101 & 204 \\
\hline Apple & iPhone 6 & D06 & On & $\mathrm{T}$ & $1920 \times 1080$ & 17 & $3264 \times 2448$ & 281 & 149 & 132 \\
\hline Apple & iPhone 6 & D15 & On & $\mathrm{T}$ & $1920 \times 1080$ & 18 & $3264 \times 2448$ & 337 & 110 & 227 \\
\hline Apple & iPhone 6 Plus & D19 & On & $\mathrm{T}$ & $1920 \times 1080$ & 19 & $3264 \times 2448$ & 428 & 169 & 259 \\
\hline Asus & Zenfone 2 Laser & D23* & On & $\mathrm{F}$ & $640 \times 480$ & 19 & $3264 \times 1836$ & 327 & 117 & 210 \\
\hline Huawei & Ascend G6-U10 & D33 & Off & $\mathrm{T}$ & $1280 \times 720$ & 19 & $2448 \times 3264$ & 239 & 84 & 155 \\
\hline Huawei & Honor 5C NEM-L51 & D30 & Off & $\mathrm{T}$ & $1920 \times 1080$ & 19 & $4160 \times 3120$ & 351 & 80 & 271 \\
\hline Huawei & P8 GRA-L09 & D28 & Off & $\mathrm{T}$ & $1920 \times 1080$ & 19 & $4160 \times 2336$ & 392 & 126 & 266 \\
\hline Huawei & P9 EVA-L09 & D03 & Off & F & $1920 \times 1080$ & 19 & $3968 \times 2976$ & 355 & 118 & 237 \\
\hline Huawei & P9 Lite VNS-L31 & D16 & Off & $\mathrm{T}$ & $1920 \times 1080$ & 19 & $4160 \times 3120$ & 350 & 115 & 235 \\
\hline Lenovo & Lenovo P70-A & D07 & Off & $\mathrm{F}$ & $1280 \times 720$ & 19 & $4784 \times 2704$ & 375 & 158 & 217 \\
\hline LG electronics & D290 & D04 & On & $\mathrm{F}$ & $800 \times 480$ & 19 & $3264 \times 2448$ & 368 & 141 & 227 \\
\hline Microsoft & Lumia 640 LTE & D17 & Off & $\mathrm{T}$ & $1920 \times 1080$ & 10 & $3264 \times 1840$ & 285 & 97 & 188 \\
\hline OnePlus & A3000 & D25 & On & T & $1920 \times 1080$ & 19 & $4640 \times 3480$ & 463 & 176 & 287 \\
\hline OnePlus & A3003 & D32 & On & $\mathrm{T}$ & $1920 \times 1080$ & 19 & $4640 \times 3480$ & 386 & 150 & 236 \\
\hline Samsung & Galaxy S III Mini GT-18190 & D26 & Off & $\mathrm{F}$ & $1280 \times 720$ & 16 & $2560 \times 1920$ & 210 & 60 & 150 \\
\hline Samsung & Galaxy S III Mini GT-18190N & D01 & Off & $\mathrm{F}$ & $1280 \times 720$ & 22 & $2560 \times 1920$ & 283 & 78 & 205 \\
\hline Samsung & Galaxy S3 GT-19300 & D11 & Off & $\mathrm{T}$ & $1920 \times 1080$ & 19 & $3264 \times 2448$ & 309 & 102 & 207 \\
\hline Samsung & Galaxy S4 Mini GT-19195 & D31 & Off & $\mathrm{T}$ & $1920 \times 1080$ & 19 & $3264 \times 1836$ & 328 & 112 & 216 \\
\hline Samsung & Galaxy S5 SM-G900F & $\mathrm{D} 27$ & Off & $\mathrm{T}$ & $1920 \times 1080$ & 19 & $5312 \times 2988$ & 354 & 100 & 254 \\
\hline Samsung & Galaxy Tab 3 GT-P5210 & D08 & Off & $\mathrm{F}$ & $1280 \times 720$ & 37 & $2048 \times 1536$ & 229 & 61 & 168 \\
\hline Samsung & Galaxy Tab A SM-T555 & D35 & Off & $\mathrm{F}$ & $1280 \times 720$ & 16 & $2592 \times 1944$ & 280 & 126 & 154 \\
\hline Samsung & Galaxy Trend Plus GT-S7580 & D22 & Off & $\mathrm{F}$ & $1280 \times 720$ & 16 & $2560 \times 1920$ & 314 & 151 & 163 \\
\hline Sony & Xperia Z1 Compact D5503 & D12 & On & $\mathrm{T}$ & $1920 \times 1080$ & 19 & $5248 \times 3936$ & 316 & 100 & 216 \\
\hline Wiko & Ridge 4G & D21 & Off & $\mathrm{T}$ & $1920 \times 1080$ & 11 & $3264 \times 2448$ & 393 & 140 & 253 \\
\hline Xiaomi & Redmi Note 3 & D24 & Off & $\mathrm{T}$ & $1920 \times 1080$ & 19 & $4608 \times 2592$ & 486 & 174 & 312 \\
\hline
\end{tabular}

DStab shows the presence or absence of digital stabilization on the acquired content, HDR indicates whether the device supports it, VR stands for video resolution and IR for image resolution

that for most Apple devices if the stabilization is present it is also enabled (the only exceptions are D9 and D13), as it is also for the Sony Xperia, D12. On the contrary, this is not true for all other devices where the in-camera digital stabilization is set off by default. In addition, Table 1 clarifies whether the device can acquire images in HDR-High Dynamic Range mode: T (True) is used if HDR is available and $\mathrm{F}$ (False) if it is not. Several additional metadata and coding statistics are collected and reported in the Appendix.

We also make available a reduced version of VISION for researchers convenience. This baseline version is composed by 16,100 images and 315 videos, both native and social, equally distributed among all the devices. In Section 5, we provide instructions for accessing the dataset. 


\subsection{Social contents}

The collected contents in VISION were also exchanged through social media platforms; in particular, for images in Nat, we provide their corresponding uploaded version on Facebook and WhatsApp. We chose to upload only natural images since, from a forensic point of view, having flat surfaces shared through social media is rather unrealistic. In addition, we shared all videos through YouTube and WhatsApp. In the rest of this Section, we explain the procedure used for uploading and downloading media contents through each social media platform.

Facebook web platform In order to exchange images via Facebook, we created two albums in which we uploaded all images belonging to Nat in high and low quality respectively (FBH and FBL from now on), as allowed by the social media platform. Indeed, as deeply explained in [19], these uploading options cause a significantly different compression strategy for the image.

For what concerns the download, we performed singleimage downloads and album downloads, although there is no difference between the resulting contents. Album download functionality was recently added to the Facebook website ${ }^{2}$ options. The one click album-download button allows downloading a zip version of each album; in each zip-file, the images are renamed by an incremental number as: $1 . j p g, 2 . j p g, \ldots n$.jpg , where $n$ is the number of images in the folder.

Since the collection of VISION lasted over a year, we exchanged data both before and after this update. We took care to provide a matching naming between the original content and the social media one: we used the SSIM index [20] as a metric to determine whether the two images depict the same content. Consequently, if the native image name is D01_I_nat_0001.jpg, its Facebook high quality counterpart will be named D01_I_natFBH_0001.jpg.

YouTube web platform All video contents were uploaded to YouTube with the Public privacy flag and collected into a playlist. During the collection of VISION, we exploited different solutions to speed-up the downloading process but maintaining the constraints of highest resolutions and no download compression. We encountered two software solutions to accomplish this goal, namely ClipGrab ${ }^{3}$ and Youtube- $d l^{4}$. Both software are freely available and can be used on several operating systems such as Unix and Windows. The main difference between the two is that the ClipGrab GUI can download one video at a time, while the youtube- $d l$ command line can download also playlists.

As an example, we provide the following youtube-dl command line call to download a playlist: ${ }^{5}$. youtube-dl -f $137+140 /$ bestvideo+best audio -o "\%(title)s.\%(ext)s" -yes-play list "device_url_playlist"

The options after the -f refers to the quality of video resolution and audio settings; here, the meaning is to choose the highest video resolution and audio quality, if not available choose the second-best pair and so on from left to right. Then with option -o we set the output video name and extension to be the YouTube video name and the default extension, i.e. mp4. For the complete documentation we advise the reader to refer to [21].

Similarly to the image naming convention, a video recorded in an outdoor scenario with a panrot movement has the following name: $\mathrm{D} 01 \mathrm{~V}_{-}$ outdoor_panrot_0001.mp4, while its YouTube counterpart will be named D01_V_outdoorYT_ panrot_0001.mp4.

WhatsApp mobile application: All native video contents and images belonging to Nat were exchanged via WhatsApp v2.17.41 using an iPhone7 A1778 with iOS v10.3.1. We decided to use the mobile-application instead of the desktop one since the latter does not compute any compression to the shared file, while the mobile one does so. We used an iPhone since it produces a media file that is less compressed than the Android one, due to WhatsApp implementation choices. In this way, we provided an equilibrate spectrum of social image contents qualities: namely high and low provided by Facebook, and medium from WhatsApp. As to the naming convention, for these files we had the same issue as in Facebook: since downloaded images are renamed, we matched images using the SSIM index.

The videos downloaded from WhatsApp follow the same name structure, (e.g., D01_V_outdoorWA _panrot_0001.mp4.

\section{Possible applications with experimental evaluations}

This dataset was created to provide a benchmark for the forensic analysis of images and videos. In this Section, we exploit all the collected contents to test the source identification technique based on the sensor pattern noise. In this scenario, the aim is to identify the source of an image or video by evaluating the correlation between the SPN fingerprint estimated from the investigated content, and the device reference fingerprint, computed from a set of images or a video taken by this device.

We tested different application scenarios:

- Image source identification (ISI), where a query image is matched with a device reference computed from a set of images taken by the device; 
- Video source identification (VSI), where a query video is matched with a device reference computed from the frames of a video taken by the device.

The identification is performed according to the classical workflow [22]: a camera fingerprint $\mathbf{K}$ is estimated from $N$ still images or video frames $\mathbf{I}^{(1)}, \ldots, \mathbf{I}^{(N)}$ captured by the source device. A denoising filter [5] is applied to each image/frame, and the noise residuals $\mathbf{W}^{(1)}, \ldots, \mathbf{W}^{(N)}$ are obtained as the difference between each frame and its denoised version. Then, the camera fingerprint estimate $\widetilde{\mathbf{K}}$ is derived by the maximum likelihood estimator [22]:

$$
\widetilde{\mathbf{K}}=\frac{\sum_{i=1}^{N} \mathbf{W}^{(i)} \mathbf{I}^{(i)}}{\sum_{i=1}^{N}\left(\mathbf{I}^{(i)}\right)^{2}} .
$$

The fingerprint of the query is estimated in the same way by the available image or video frames. Then, the Peak to Correlation Energy (PCE) between the reference and the query pattern is computed and compared to a threshold [23]: if the PCE is higher than the threshold, then it is decided that the query content has been acquired by the reference device.

\subsection{Image source identification}

In this scenario, the reference SPN for each device is estimated using 100 still flat field images. Then, we run four experiments using natural, WhatsApp, Facebook highquality, and Facebook low-quality images as queries. In all experiments, we consider for each device 100 matching cases (images from the same device) and the same number of mismatching cases (images randomly chosen from other devices). The achieved results are reported using ROC curves that plot true positive rate against false positive rate (see Fig. 3). The overall performance are summarized in Table 2 where, for each experiment, we also reported the dataset path of the query images and the Area Under Curve. ID_Brand_Model stands for any of the available device e.g., D03_Huawei_P9.

\subsection{Video source identification}

Here, the source of a test video is determined based on references estimated from a flat-field video. In particular, the reference SPN for each device is estimated from the first 100 frames of a flat video. Then, three experiments are performed using natural, YouTube and WhatsApp videos as queries, respectively. The fingerprint of each tested video is estimated from the first 100 frames. We consider for each device all available matching cases (videos from the same device) and the same number of mismatching cases (videos randomly chosen from other devices). The achieved results are reported in Fig. 4, where only nonstabilized cameras are analyzed, and in Fig. 5, where all

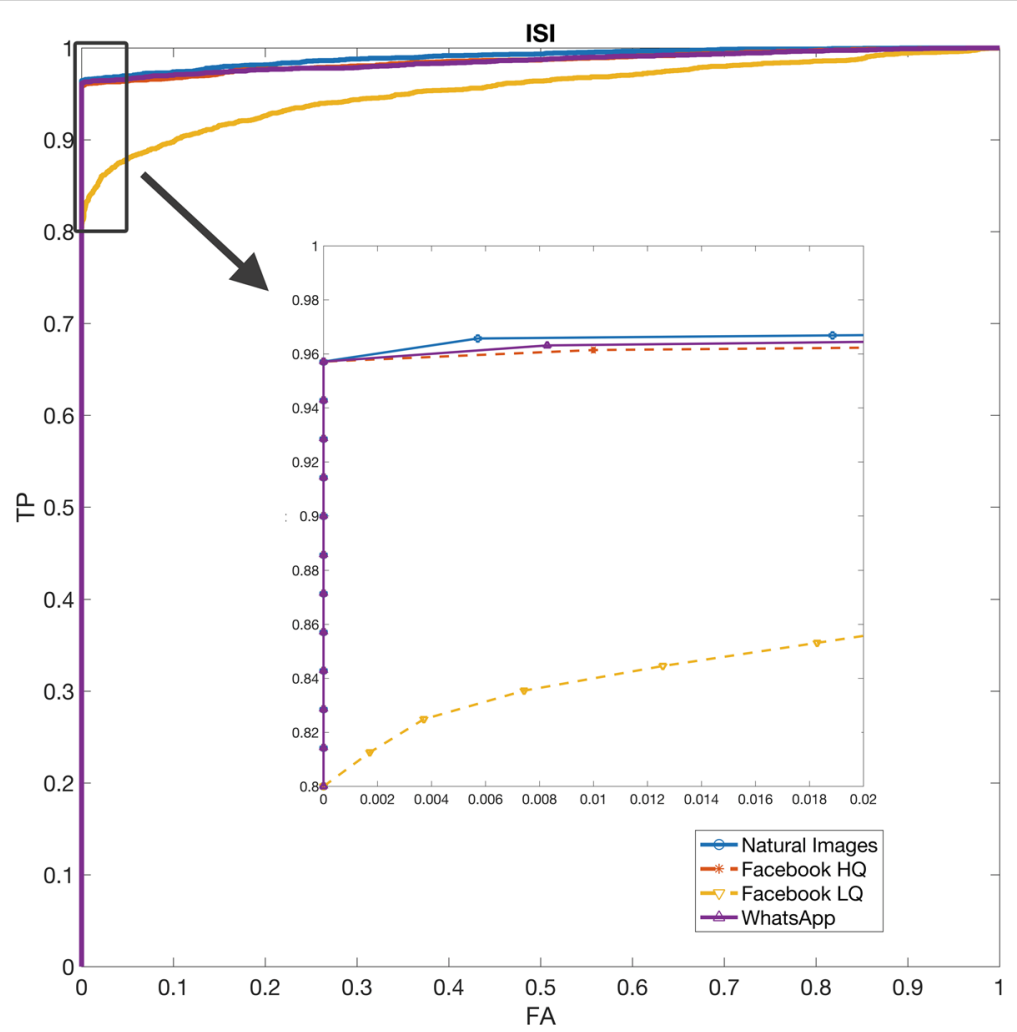

Fig. 3 (Best viewed in colors) ISI performance on Native, Facebook (HQ and LQ), and WhatsApp images using flat field references 
Table 2 Performance of image source identification in growing difficulty scenarios

\begin{tabular}{lll}
\hline Experiment & Test Path & AUC \\
\hline 1 & ID_Brand_Model/images/nat & 0.9906 \\
2 & ID_Brand_Model/images/natWA & 0.9860 \\
3 & ID_Brand_Model/images/natFBH & 0.9859 \\
4 & ID_Brand_Model/images/natFBL & 0.9544 \\
\hline
\end{tabular}

devices in the dataset are considered. This experiment shows that performance of VSI strongly drop when digitally stabilized videos are involved. In Table 3, we briefly summarize for each test the paths in the dataset of tested videos and the Area Under Curve values obtained with and without stabilized videos.

For in-camera stabilized videos, possible solutions are still under development, as the one proposed in [24]. Anyway the solution in [24] is proved to be effective only on third party (out-camera) digital stabilization (ffmpeg), and when a non-stabilized video is available as reference. Unfortunately, most of the considered devices enforce incamera digital stabilization, without an option to turn it off in the standard camera application.

\subsection{Image vs video SPN fingerprint}

In the research community, ISI and VSI applications are separately studied so that there is still no better way to perform image and video source identification for the same

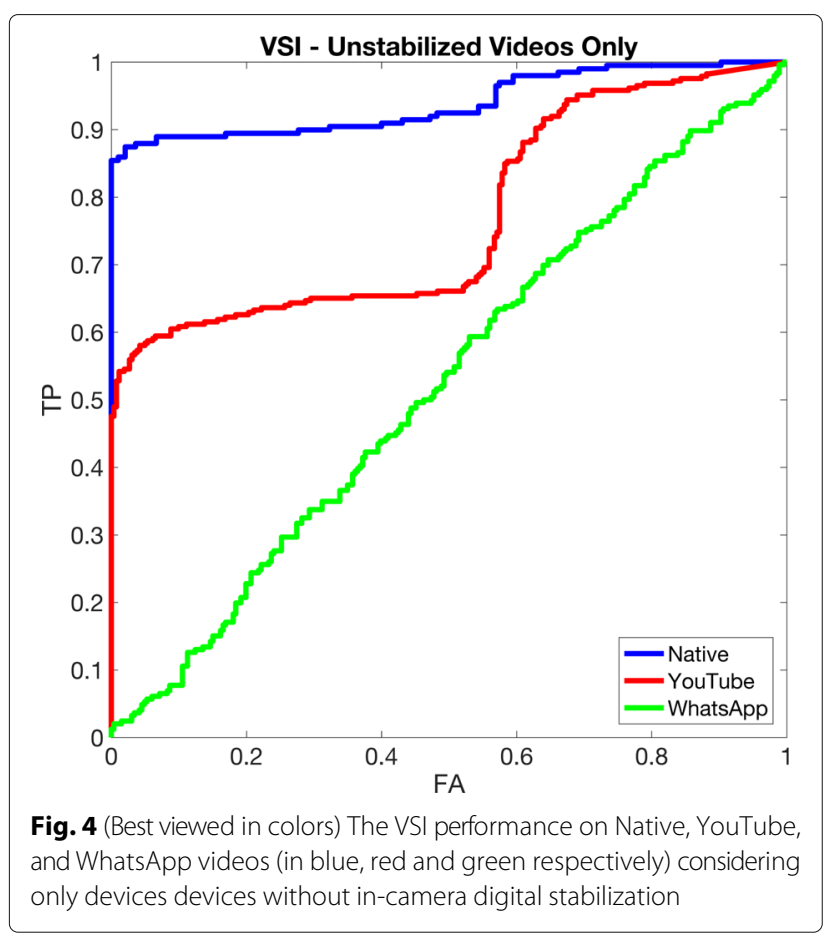

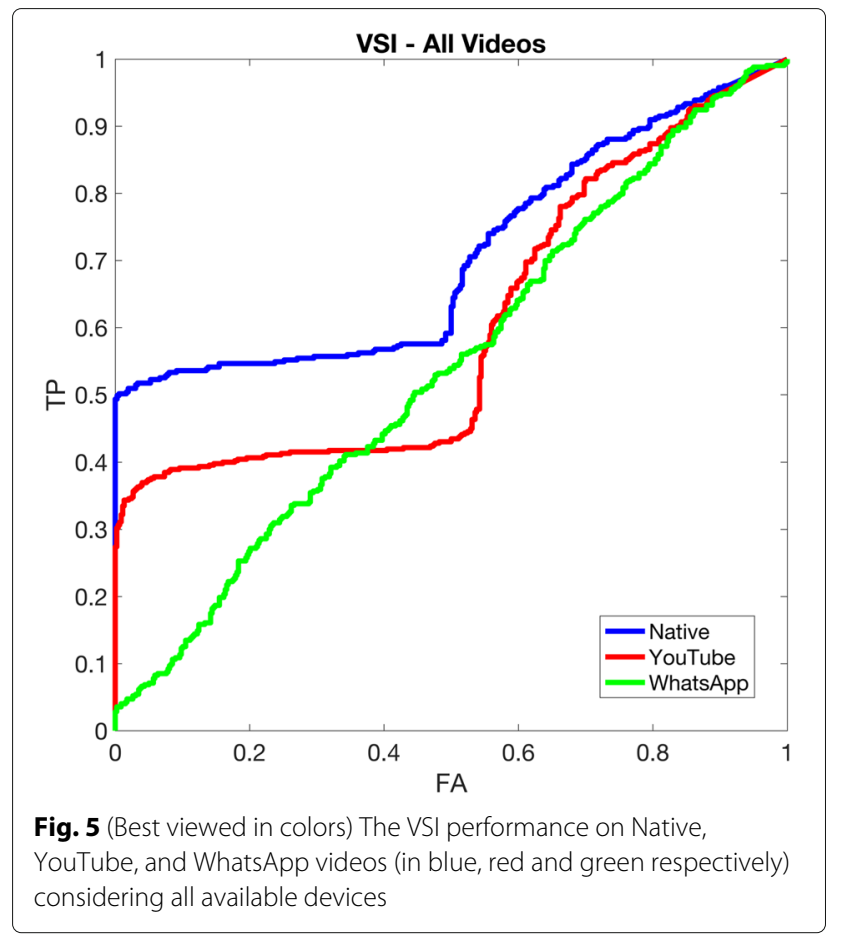

device than computing two different reference SPNs, one for still images and one for videos, respectively.

A first step towards an integration of these cases is a hybrid source identification (HSI) approach that exploits still images for estimating the fingerprint that will be used to verify the source of a video, as proposed in [4]. Authors of [4] investigate the geometrical relation between image and video acquisition processes. Indeed, even if the sensor is the same, videos are usually acquired at a much lower resolution than images: top-level smartphones reach $4 \mathrm{~K}$ video resolution at most (8 megapixels per frame), but can easily capture 20-megapixel images. To achieve that, in video recording, a central crop is carried so to adapt the sensor size to the desired aspect ratio (commonly 16:9), then the selected pixels are scaled to match the desired video resolution. As a direct consequence, the fingerprints extracted from images and videos cannot be directly compared and most of the times, because of cropping, it is not sufficient to just scale them to the same resolution. Instead, image-based and video-based fingerprints are linked by the cropping and scaling factors between image and video sensor portion, that usually change across different device models.

With the aim of facilitating researchers exploring the HSI framework within the VISION dataset, we provide the cropping and scaling factor for several devices contained therein. For simplicity, we limit to non-stabilized devices; the hybrid analysis for stabilized devices is even more complex, and is one of the future research scopes this dataset has been built for. In order to estimate 
Table 3 Dataset paths for VSI experiments

\begin{tabular}{lll}
\hline Experiment & Test path & AUC \\
\cline { 3 - 3 } 1 & All Videos & \\
& ID_Brand_Model/videos/flat & Unstab. Videos \\
& ID_Brand_Model/videos/indoor & 0.7069 \\
& ID_Brand_Model/videos/outdoor & \\
& ID_Brand_Model/videos/flatYT & \\
& ID_Brand_Model/videos/indoorYT & 0.9394 \\
& ID_Brand_Model/videos/outdoorYT & 0.6032 \\
& ID_Brand_Model/videos/flatWA & \\
& ID_Brand_Model/videos/flatWA & \\
& ID_Brand_Model/videos/flatWA & 0.5262 \\
\hline
\end{tabular}

cropping and scaling factors, for each device we estimated the video and image references from the videos contained in ID_Brand_Model/videos/flat and from the images in ID_Brand_Model/images/flat, respectively. Specifically, we estimated each image reference fingerprint from 100 flat field images and each video reference fingerprint from 100 frames of a flat field video. The cropping and scaling factors are estimated by a brute force search, as suggested in [25]. In Table 4 we report the scaling factor and the corresponding cropping corner (upper-left corner along $x$ and $y$ axes) yielding the maximum PCE for each examined device. We consider the parameter search unsuccessful if the obtained maximum PCE is lower than 50 (denoted by "n.a." in Table 4). For instance, with the device $D 11$ an image fingerprint should be scaled by a factor 0.59 and then cropped on the upper left side of 307 pixels along the $y$ axis to match the video fingerprint (the right and down cropping are derived by the corresponding video size). D13 is a pretty unique case in which the full frame is applied for videos and the left (and right) cropping of 160 pixels is applied to capture images. We put a -160 meaning that the video frame is cropped by 160 pixel to capture images. Finally, we notice that we were not able to register the fingerprints for the devices $D 21$ and $D 26$ by means of the presented techniques. A deeper analysis of the registration techniques is still an open topic.

\section{Conclusions}

In this paper, we propose a new image and video dataset useful for benchmarking multimedia forensic tools. We collected thousands of images and videos from portable devices of most famous brands, including those featuring in-camera digital stabilization. We also prepared the "social version" of most contents, by uploading and downloading them to/from well-known social media platforms, namely Facebook, YouTube and WhatsApp.

We showed examples of some popular applications that would benefit from the proposed dataset, such as the video source identification, for which there are no sizeable benchmarks available in the research community. Furthermore, we showed how this dataset allows the exploration of new forensic opportunities such as comparing camera reference fingerprints estimated from still images and from videos. The whole dataset is made available ${ }^{6}$ to the research community, along with a guide that clarifies its structure and several csv files containing technical information.

Although VISION is a huge collection of media contents, we believe that there is space for future improvements, indeed we are currently working to extend VISION with more videos, by means of a mobile application (MOSES [26]) from which videos can be captured and uploaded directly to our servers, following the main concept and guidelines of VISION. In conclusion, VISION

Table 4 Estimated Cropping and Scaling factors for non stabilized videos

\begin{tabular}{|c|c|c|c|c|c|c|c|c|c|c|}
\hline ID & D01 & D03 & D07 & D08 & D09 & D11 & D13 & D16 & D17 & D21 \\
\hline Scaling & 0.5 & 0.48 & 0.27 & 1 & 0.61 & 0.59 & 1 & 0.46 & 0.59 & п.а. \\
\hline Cropping $[x y]$ & [0 228] & {$\left[\begin{array}{ll}0 & 372\end{array}\right]$} & {$\left[\begin{array}{ll}0 & 7\end{array}\right]$} & [408 354] & [227 411] & [0 307] & {$\left[\begin{array}{lll}-160 & 0\end{array}\right]$} & [8 396] & {$\left[\begin{array}{ll}0 & 1\end{array}\right]$} & n.a. \\
\hline ID & D24 & D26 & D27 & D28 & D30 & D31 & D32 & D33 & D35 & D22 \\
\hline Scaling & 0.5 & n.a. & 0.5 & 0.36 & 0.47 & 0.46 & 0.59 & 0.52 & 0.39 & 0.49 \\
\hline Cropping $[x y]$ & [0 240] & n.a. & [0 228] & {$\left[\begin{array}{ll}0 & 0\end{array}\right]$} & [39 10] & [9 397] & {$\left[\begin{array}{ll}0 & 0\end{array}\right]$} & [464 693] & [0 306] & [0 246] \\
\hline
\end{tabular}


provides the contents needed to assess the performance of next generation image and video forensic tools.

\section{Endnotes}

${ }^{1}$ Not all the videos were exchanged through social media platforms. The technical details are explained in the Appendix

${ }^{2}$ Facebook website on March 2017.

${ }^{3}$ ClipGrab v3.6.3 - www.clipgrab.org

${ }^{4}$ youtube-dl v2017.03.10 - http://rg3.github.io/youtube$\mathrm{dl} /$

${ }^{5}$ We recommend downloading less than 20 videos at a time due to the YouTube policy.

${ }^{6}$ https://lesc.dinfo.unifi.it/en/datasets

${ }^{7}$ Note that the Rotation tag, related to the correspondent video standard, of each video is reported in the additional material released together with the dataset.

\section{Appendix}

In this section, we provide more detailed information on the VISION dataset. First of all, in Appendix Table 5, we review the version of the software/firmware of each device, along with the number of native images and videos (in columns \#natOrigin and \#vOrigin) and their social counterparts (in columns \#natSocial, \#vSocial). We would like the reader to notice that the amount of social media videos should be two times the original amount, since we uploaded all the videos in YouTube and WhatsApp. The reader can easily see that this is not true for some devices, indeed we removed 30 videos from the social folders because of issues due to the uploading system used by YouTube and WhatsApp. We acquired videos from each device using the landscape-mode, but a small amount of the overall acquisitions were captured in portrait mode. Unfortunately this was an issue with the YouTube uploading, because in this case, the video is modified by black padding: this raised a consistency problem and we decided to remove these contents. On the other hand, WhatsApp was not affected by this kind of problems, and the same portrait-mode videos were uploaded and downloaded correctly. Unfortunately, we could not upload 4 videos with the WhatsApp iOS application, and so far did not understand the reason causing this problem.

The devices affected by these problems were the D34 in which we exluded 14 videos, the D01 with 6 videos missing, the D08 with 4 files, the D06 with 2 files and one file from devices D04, D21, D22, and D33. Detailed information are given, in form of multiple CSV file, with the current Dataset.

In Appendix Table 6 we report for each device some statistics computed over all native images by means of
Exiftool 10.10. In detail, the metadata tags that can be extracted with Exiftool are the Lens Model, ISO, Aperture, Flash, Focal Length, Image Size (represented in column Image Resolution) and Orientation.

All images were acquired in the JPEG format using the encoder JPEG old-style, and for most devices the color sub sampling is set to $\mathrm{YCbCr}$ 4:2:0 (2,2), while D07 and D11 use $Y C b C r$ 4:2:2 $(2,1)$ and only D28 supports both sub samplings.

The columns create/modify date and GPS present are counters: the first one counts the number of images in which the metadata create date and modify date are not identical. The second refers to the metadata GPS Position and counts the number of images in which this tag is not empty.

In Appendix Table 6, we collected information related to the Lens specifics such as the focal length, the aperture value or the ISO. It is worth noting that the Lens model tag is present only for Apple devices, and specifies which camera is used and some features of the lens. The ISO column contains a range of values for each device, that is the minimum and the maximum ISO value observed in the images metadata from that device. The Flash column lists all encountered values, indeed for some devices such as D05, we have images acquired with Flash in auto mode, off mode, fired, or not fired mode. In case of devices D13 and D20, namely iPad 2 and iPad mini, the flash function does not exists, since these devices are not equipped with a flash.

The Image Size tag is present in column image resolution and reports the resolution as width $\times$ height; let us note that in devices D24 and D33 the image Orientation tag is not always present, when needed it is derived from their resolution in order to distinguish between landscape and portrait acquisitions such as $4608 \times 2592$ and $2592 \times 4608$ for D24. In all the remaining devices, each image is stored in landscape mode, that is horizontally $(\mathrm{H})$; if the image was actually acquired with a different orientation, this is reflected in the Orientation tag, which may contain the values: Rotation 180 (R 180), Rotation 90 Clock-Wise (R $90 \mathrm{CW}$ ) or Rotation 270 Clock-Wise (R $270 \mathrm{CW}$ ).

From a forensics point of view the create/modify date is very interesting: in Appendix Table 6 the reader can see that for all Apple devices in this Dataset, the Create Date and Modify Date tags are different (when available), meaning that Apple devices store into Create Date the moment in which the photo shoot is computed and in Modify Date the moment in which the image is stored, that is, typically, a few seconds later. All the other devices set these tags to the same initial value.

In Appendix Table 7, we report for each device some statistical information computed over the recorded videos. The metadata tags gathered from the native videos by means of Exiftool were file type, major brand, video frame 
Table 5 Devices featured in VISION

\begin{tabular}{|c|c|c|c|c|c|c|c|}
\hline Brand & Model & ID & Software/firmware & \#vOrigin & \#vSocial & \#natOrigin & \#natSocia \\
\hline Apple & iPad 2 & D13 & iOS 7.1.1 & 16 & 32 & 171 & 513 \\
\hline Apple & iPad mini & D20 & iOS 8.4 & 16 & 32 & 159 & 477 \\
\hline Apple & iPhone 4 & D09 & iOS 7.1.2 & 19 & 38 & 217 & 651 \\
\hline Apple & iPhone 4S & D02 & iOS 7.1.2 & 13 & 26 & 204 & 612 \\
\hline Apple & iPhone 4S & D10 & iOS 8.4.1 & 15 & 30 & 178 & 534 \\
\hline Apple & iPhone 5 & D29 & iOS 9.3.3 & 19 & 38 & 224 & 672 \\
\hline Apple & iPhone 5 & D34 & iOS 8.3 & 32 & 50 & 204 & 612 \\
\hline Apple & iPhone 5c & D05 & iOS 10.2.1 & 19 & 38 & 350 & 1050 \\
\hline Apple & iPhone 5c & D14 & iOS 7.0.3 & 19 & 38 & 209 & 627 \\
\hline Apple & iPhone 5c & D18 & iOS 8.4.1 & 13 & 26 & 204 & 612 \\
\hline Apple & iPhone 6 & D06 & iOS 8.4 & 17 & 32 & 132 & 396 \\
\hline Apple & iPhone 6 & D15 & iOS 10.1.1 & 18 & 36 & 227 & 681 \\
\hline Apple & iPhone 6 Plus & D19 & iOS 10.2.1 & 19 & 38 & 259 & 777 \\
\hline Asus & Zenfone 2 Laser & D23* & - & 19 & 38 & 210 & 630 \\
\hline Huawei & Ascend G6-U10 & D33 & - & 19 & 37 & 155 & 465 \\
\hline Huawei & Honor 5C NEM-L51 & D30 & Android 6.0/NEM-L51C432B120 & 19 & 38 & 271 & 813 \\
\hline Huawei & P8 GRA-L09 & D28 & Android 6.0/GRA-L09C55B330 & 19 & 38 & 266 & 798 \\
\hline Huawei & P9 EVA-L09 & D03 & Android 6.0/EVA-L09C55B190 & 19 & 38 & 237 & 711 \\
\hline Huawei & P9 Lite VNS-L31 & D16 & Android 6.0/NNS-L31C02B125 & 19 & 38 & 235 & 705 \\
\hline Lenovo & Lenovo P70-A & D07 & - & 19 & 38 & 217 & 651 \\
\hline LG electronics & D290 & D04 & - & 19 & 37 & 227 & 681 \\
\hline Microsoft & Lumia 640 LTE & D17 & Windows Phone & 10 & 20 & 188 & 564 \\
\hline OnePlus & A3000 & D25 & Android 7.0/NRD90M 15 dev-keys & 19 & 38 & 287 & 861 \\
\hline OnePlus & A3003 & D32 & Android 7.0/NRD90M 138 dev-keys, & 19 & 38 & 236 & 708 \\
\hline- & - & - & NRD90M 18 dev-keys & - & - & - & - \\
\hline Samsung & Galaxy S III Mini GT-18190 & D26 & I8190XXAMG4 & 16 & 32 & 150 & 450 \\
\hline Samsung & Galaxy S III Mini GT-18190N & D01 & I8190NXXAML1, I8190NXXALL6 & 22 & 38 & 205 & 615 \\
\hline Samsung & Galaxy S3 GT-19300 & D11 & - & 19 & 38 & 207 & 621 \\
\hline Samsung & Galaxy S4 Mini GT-19195 & D31 & 19195XXUCNK1 & 19 & 38 & 216 & 648 \\
\hline Samsung & Galaxy S5 SM-G900F & D27 & Android 6.0.1/G900FXXS1CQAA & 19 & 38 & 254 & 762 \\
\hline Samsung & Galaxy Tab 3 GT-P5210 & D08 & P5210XXUBNK2 & 37 & 70 & 168 & 504 \\
\hline Samsung & Galaxy Tab A SM-T555 & D35 & T555XXU1AOE9 & 16 & 32 & 154 & 462 \\
\hline Samsung & Galaxy Trend Plus GT-S7580 & D22 & S7580XXUBOA1 & 16 & 31 & 163 & 489 \\
\hline Sony & Xperia Z1 Compact D5503 & D12 & 14.5.A.0.270_6_f100000f & 19 & 38 & 216 & 648 \\
\hline Wiko & Ridge 4G & D21 & - & 11 & 21 & 253 & 759 \\
\hline Xiaomi & Redmi Note 3 & D24 & Android 6.0.1/MMB29M & 19 & 38 & 312 & 936 \\
\hline- & - & - & V8.1.1.0.MHOMIDI release-keys & - & - & - & - \\
\hline
\end{tabular}

rate, media duration, audio channels, audio sample rate, image size, and rotation ${ }^{7}$.

In order to make Appendix Table 7 clearer, we used video resolution instead of the tag Image Size, and we did not report the tags related to the audio acquisition, although they will be described in the following paragraphs.
All videos were acquired using the video encoder H.264/avc1 and mp4a for encoding audio. We remark that, for the D23 device videos were not captured at the maximum resolution available, as opposed to all other acquisitions. Similarly to Appendix Table 6, we included in Appendix Table 7 the columns create/modify date and 


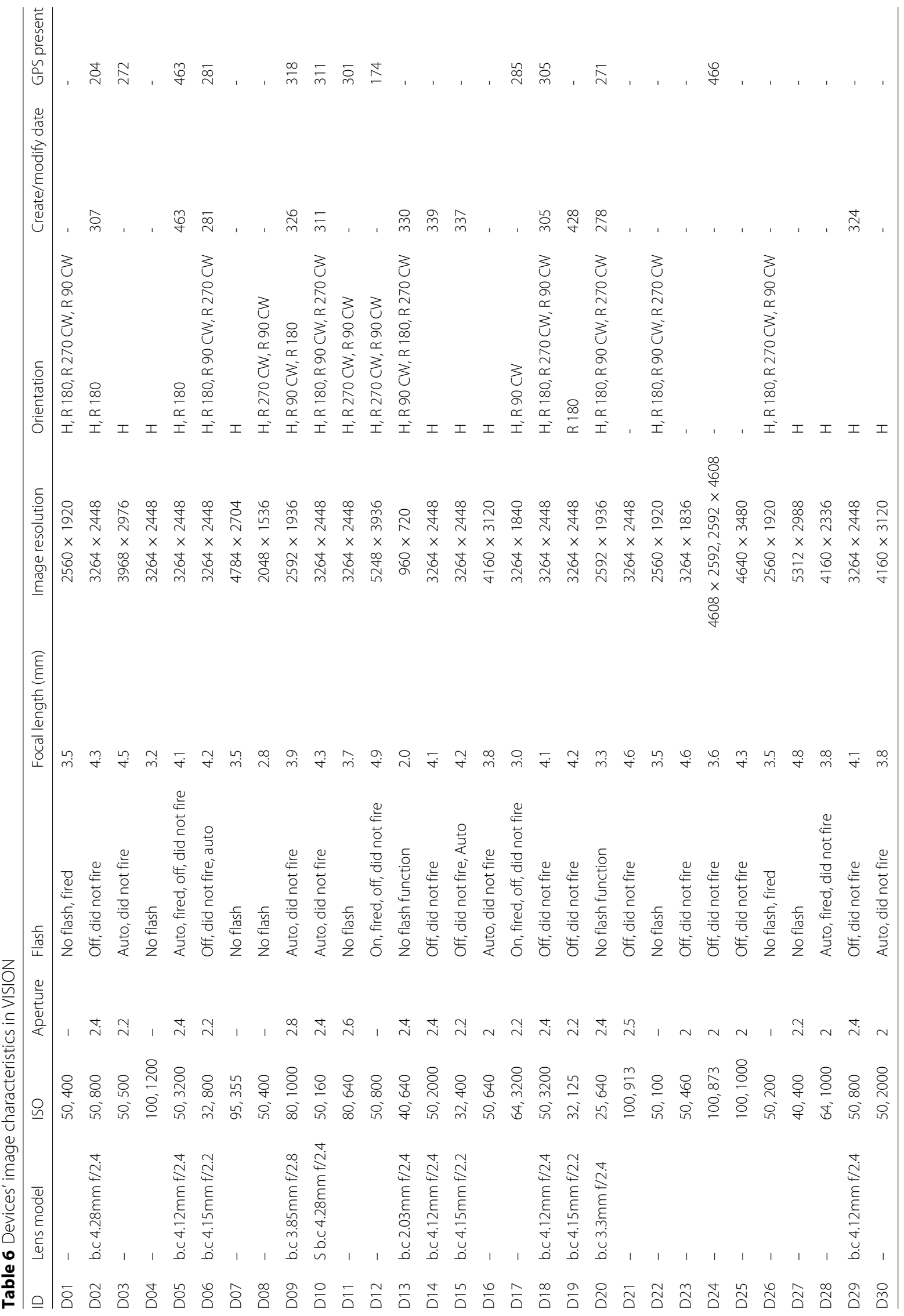




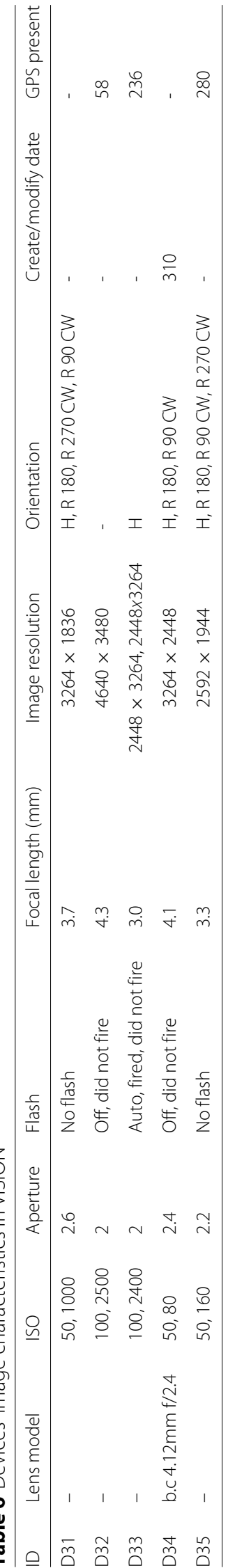


Table 7 Devices' video characteristics in VISION

\begin{tabular}{|c|c|c|c|c|c|c|c|c|}
\hline ID & File type & Video format & Frame rate & Media duration & Video resolution & Rotation & $\begin{array}{l}\text { Create/modify } \\
\text { date }\end{array}$ & $\begin{array}{l}\text { GPS } \\
\text { present }\end{array}$ \\
\hline D01 & MP4 & MP4 Base Media v1 & $28.986,30.233$ & $0: 01: 08,0: 01: 13$ & $1280 \times 720$ & 0,90 & - & - \\
\hline D02 & MOV & Apple QuickTime (.MOV/QT) & $24.009,29.97$ & $0: 00: 59,0: 01: 12$ & $1920 \times 1080$ & 0,180 & 13 & 11 \\
\hline D03 & MP4 & MP4 V2 ISO 14496-14 & $30.011,30.033$ & $0: 01: 10,0: 01: 17$ & $1920 \times 1080$ & 0,180 & - & - \\
\hline D04 & MP4 & MP4 Base Media v1 & $26.038,30.024$ & $0: 01: 10,0: 01: 14$ & $800 \times 480$ & 0 & - & - \\
\hline D05 & MOV & Apple QuickTime (.MOV/QT) & $25.008,29.973$ & 0:00:26.78, 0:01:18 & $1920 \times 1080$ & 0,180 & 19 & 19 \\
\hline D06 & MOV & Apple QuickTime (.MOV/QT) & $30.006,30.006$ & $0: 01: 06,0: 01: 11$ & $1920 \times 1080$ & 0,90 & 17 & 17 \\
\hline D07 & $3 G P$ & 3GPP Media (.3GP) Release 4 & $22.694,30.004$ & $0: 01: 11,0: 01: 14$ & $1280 \times 720$ & 0,180 & - & - \\
\hline D08 & MP4 & MP4 Base Media v1 & $24.016,29.686$ & $0: 01: 01,0: 01: 11$ & $1280 \times 720$ & $0,90,270$ & - & - \\
\hline D09 & MOV & Apple QuickTime (.MOV/QT) & $28.621,29.969$ & 0:01:09, 0:01:16 & $1280 \times 720$ & 0 & 19 & 17 \\
\hline D10 & MOV & Apple QuickTime (.MOV/QT) & $24.01,29.97$ & $0: 01: 10,0: 01: 13$ & $1920 \times 1080$ & 0 & 15 & 15 \\
\hline D11 & MP4 & MP4 Base Media v1 & $29.978,30.006$ & $0: 01: 11,0: 01: 17$ & $1920 \times 1080$ & 0 & - & 3 \\
\hline D12 & MP4 & MP4 V2 ISO 14496-14 & $29.904,29.982$ & $0: 01: 10,0: 01: 24$ & $1920 \times 1080$ & 0 & - & - \\
\hline D13 & MOV & Apple QuickTime (.MOV/QT) & $28.727,29.967$ & $0: 01: 09,0: 01: 13$ & $1280 \times 720$ & 0 & 16 & - \\
\hline D14 & MOV & Apple QuickTime (.MOV/QT) & $29.973,29.973$ & $0: 01: 12,0: 01: 13$ & $1920 \times 1080$ & 0 & 19 & - \\
\hline D15 & MOV & Apple QuickTime (.MOV/QT) & $29.983,29.984$ & $0: 01: 02,0: 01: 05$ & $1920 \times 1080$ & 0 & 18 & - \\
\hline D16 & MP4 & MP4 V2 ISO 14496-14 & $30.167,30.205$ & $0: 01: 10,0: 01: 14$ & $1920 \times 1080$ & 0,180 & - & - \\
\hline D17 & MP4 & MP4 v2 ISO 14496-14 & $30.008,30.008$ & $0: 01: 10,0: 01: 11$ & $1920 \times 1080$ & 0 & - & - \\
\hline D18 & MOV & Apple QuickTime (.MOV/QT) & $24.003,29.973$ & $0: 01: 09,0: 01: 12$ & $1920 \times 1080$ & 0 & 13 & 13 \\
\hline D19 & MOV & Apple QuickTime (.MOV/QT) & $29.983,30.0$ & $0: 01: 10,0: 01: 15$ & $1920 \times 1080$ & 0,180 & 19 & - \\
\hline D20 & MOV & Apple QuickTime (.MOV/QT) & $29.576,29.972$ & $0: 01: 10,0: 01: 12$ & $1920 \times 1080$ & 0 & 16 & 7 \\
\hline D21 & MP4 & MP4 V2 ISO 14496-14 & $18.893,29.246$ & $0: 01: 07,0: 01: 26$ & $1920 \times 1080$ & 0 & - & - \\
\hline D22 & MP4 & MP4 Base Media v1 & $30.03,30.034$ & $0: 01: 10,0: 01: 20$ & $1280 \times 720$ & 0 & - & - \\
\hline D23* & MP4 & MP4 v2 ISO 14496-14 & $29.891,29.903$ & $0: 01: 09,0: 01: 14$ & $640 \times 480$ & 0 & - & - \\
\hline D24 & MP4 & MP4 v2 ISO 14496-14 & $20.215,30.062$ & $0: 01: 07,0: 01: 13$ & $1920 \times 1080$ & 0 & - & 11 \\
\hline D25 & MP4 & MP4 v2 ISO 14496-14 & $29.999,30.01$ & $0: 01: 10,0: 01: 17$ & $1920 \times 1080$ & 0,180 & - & - \\
\hline D26 & MP4 & MP4 Base Media v1 & $29.262,30.237$ & $0: 01: 11,0: 01: 14$ & $1280 \times 720$ & 0 & - & - \\
\hline D27 & MP4 & MP4 v2 ISO 14496-14 & $29.97,30.006$ & 0:00:25.54, 0:01:16 & $1920 \times 1080$ & 0 & - & - \\
\hline D28 & MP4 & MP4 v2 ISO 14496-14 & $29.636,29.886$ & $0: 01: 09,0: 01: 16$ & $1920 \times 1080$ & 0,180 & - & - \\
\hline D29 & MOV & Apple QuickTime (.MOV/QT) & $24.003,29.973$ & $0: 01: 10,0: 01: 15$ & $1920 \times 1080$ & 0 & 19 & - \\
\hline D30 & MP4 & MP4 v2 ISO 14496-14 & $30.14,30.157$ & $0: 01: 08,0: 01: 17$ & $1920 \times 1080$ & 0,180 & - & - \\
\hline D31 & MP4 & MP4 Base Media v1 & $29.927,30.013$ & $0: 01: 12,0: 01: 16$ & $1920 \times 1080$ & 0 & - & - \\
\hline D32 & MP4 & MP4 v2 ISO 14496-14 & $29.898,30.01$ & $0: 01: 09,0: 01: 16$ & $1920 \times 1080$ & 0 & - & 2 \\
\hline D33 & MP4 & MP4 Base Media v1 & $24.967,30.03$ & $0: 01: 10,0: 01: 14$ & $1280 \times 720$ & 0,90 & - & 19 \\
\hline D34 & MOV & Apple QuickTime (.MOV/QT) & $24.003,29.973$ & $0: 01: 08,0: 01: 32$ & $1920 \times 1080$ & 0,90 & 32 & - \\
\hline D35 & MP4 & MP4 v2 ISO 14496-14 & $29.873,30.012$ & $0: 01: 10,0: 01: 13$ & $1280 \times 720$ & 0 & - & 16 \\
\hline
\end{tabular}

GPS present. The former counts the number of videos in which metadata create date and modify date are not identical, while the latter counts the number of videos in which the GPS location tag is not empty.

We highlight that the values in the create/modify date column are different than 0 for Apple devices only: indeed, in these devices the create date differs from the modify date by the duration of the video.
All Apple devices store videos with the Apple QuickTime container (.MOV extension), and most of Android devices store video as MP4 using the H.264 encoder in two versions: MP4 Base v1, ISO 14496-12 or MP4 Base v2, ISO 14496-14. It is worth mentioning that the Lenovo device (D07) encodes videos in H.264 but uses the container 3GP. Almost all videos last more than one minute, with the exception of a few in devices D02, D05, 
and D27 where the shortest video duration is $25 \mathrm{sec}$ onds. Most devices record videos at a frame rate of 24 fps or more; as exceptions we have some videos from D07, D21 and D24.

As to the characteristics of the encoded audio, as a general distinction Apple devices acquire audio using one channel at sample rate of $44100 \mathrm{bit} / \mathrm{s}$, whereas Android devices usually acquire two audio channels at 48000 bit/s. The Microsoft device (D17) uses one audio channel at $48000 \mathrm{bit} / \mathrm{s}$, like some Samsung Devices, namely: Galaxy S III Mini (D01, D26) and the Galaxy Trend Plus (D22).

\section{Abbreviations}

Exif: Exchangeable image file format; $\mathrm{HSI}$ : Hybrid source identification; IS: Image source identification; MF: Multimedia forensics; PCE: Peak to correlation energy; ROC: Receiver operating characteristic; SMP: Social media platform: SPN: Sensor pattern noise; VSI: Video source identification

\section{Availability of data and materials}

As stated in Section 5, the whole dataset will be made available at no cost to the research community at the following web address https://lesc.dinfo.unifi. it/en/datasets, with the goal of providing the content needed to assess the performance of next generation of image and video forensic tools. In addition to the dataset we release a guide to the dataset structure and several csv files containing names and technical information, such as metadata tags, of all the collected media.

\section{Authors' contributions}

DS contributed to acquisition of data (especially for obtaining the social-network version of videos), prepared all the statistics about data and corresponding metadata, and drafted part of the manuscript. MF took care of writing code, analyse and present results for the experimental section about image and video source identification. MI contributed to drafting the paper and writing code, and analysed the relationships between sensor pattern noise in images and videos. OAS carried out most of the data acquisition, checked the gathered material and contributed to obtaining the social-network version of images. AP conceived the study, participated to its design and coordination, and helped to draft the manuscript. All authors read and approved the final manuscript.

Ethics approval and consent to participate

Not applicable.

\section{Consent for publication}

Not applicable.

\section{Competing interests}

The authors declare that they have no competing interests.

\section{Publisher's Note}

Springer Nature remains neutral with regard to jurisdictional claims in published maps and institutional affiliations.

\footnotetext{
Author details

${ }^{1}$ Department of Information Engineering, University of Florence, Via di S. Marta, 3, 50139 Florence, Italy. ${ }^{2}$ FORLAB, Multimedia Forensics laboratory, PIN Scrl, Piazza G. Ciardi, 25, 59100 Prato, Italy. ${ }^{3}$ Department of electronic Media, Saudi Electronic University, Abi Bakr As Sadiq Rd, Riyadh, 11673, Saudi Arabia.
}

Received: 10 May 2017 Accepted: 18 September 2017

Published online: 03 October 2017

\section{References}

1. Statista Inc., Statista. http://www.statista.com/statistics/263437/globalsmartphone-sales-to-end-users-since-2007/. Accessed 22 Sept 2017

2. A De Rosa, A Piva, M Fontani, M luliani, in 2014 International Carnahan Conference on Security Technology (ICCST). Investigating multimedia contents (IEEE, Rome, 2014), pp. 1-6

3. A Piva, An overview on image forensics. ISRN Signal Proc. 2013 496701-22 (2013)

4. M luliani, M Fontani, D Shullani, A Piva, A hybrid approach to video source identification. arXiv:1705.01854[Cs.MM] (2017)

5. J Lukas, J Fridrich, M Goljan, Digital camera identification from sensor pattern noise. IEEE Trans. Inf. Forensic Secur. 1(2), 205-214 (2006)

6. W Van Houten, Z Geradts, Source video camera identification for multiply compressed videos originating from youtube. Digit. Investig. 6(1), 48-60 (2009)

7. M Chen, J Fridrich, M Goljan, J Lukáš, in Proc. of SPIE 6515 Electronic Imaging 2007. Source digital camcorder identification using sensor photo response non-uniformity, (2007), pp. 65051-65051. International Society for Optics and Photonics

8. W-H Chuang, H Su, M Wu, in IEEE International Conference on Image Processing (ICIP). Exploring compression effects for improved source camera identification using strongly compressed video (IEEE, Brussels, 2011), pp. 1953-1956

9. S Chen, A Pande, K Zeng, P Mohapatra, Live video forensics: source identification in lossy wireless networks. IEEE Trans. Inf. Forensic Secur. 10(1), 28-39 (2015)

10. G Schaefer, M Stich, in Proc. SPIE 5307 Electronic Imaging 2004. Ucid: an uncompressed color image database, (2003), pp. 472-480. International Society for Optics and Photonics

11. T Gloe, R Böhme, The Dresden image database for benchmarking digital image forensics. J. Digit. Forensic Pract. 3(2-4), 150-159 (2010)

12. T Gloe, R Böhme, in Proceedings of the 25th Symposium On Applied Computing (ACM SAC 2010). The 'Dresden Image Database' for benchmarking digital image forensics, vol. 2 (ACM New York, Sierre, 2010), pp. 1585-1591

13. D-T Dang-Nguyen, C Pasquini, V Conotter, G Boato, in Proceedings of the 6th ACM Multimedia Systems Conference. MMSys '15. Raise: a raw images dataset for digital image forensics (ACM, New York, 2015), pp. 219-224

14. D Vázquez-Padín, F Pérez-González, in 2011 IEEE International Workshop on Information Forensics and Security. Prefilter design for forensic resampling estimation (IEEE, Iguacu Falls, 2011), pp. 1-6

15. G Qadir, S Yahaya, ATS Ho, in Proceedings of the IET IPR 2012, 3-4 July, London. Surrey University Library for Forensic Analysis (SULFA), (2012)

16. L D’Amiano, D Cozzolino, G Poggi, L Verdoliva, in Multimedia \& Expo Workshops (ICMEW), 2015 IEEE International Conference On. Video forgery detection and localization based on 3d patchmatch (IEEE, Turin, 2015), pp. 1-6

17. Ol Al-Sanjary, AA Ahmed, G Sulong, Development of a video tampering dataset for forensic investigation. Forensic Sci. Int. 266, 565-572 (2016)

18. F Bertini, R Sharma, A lannı, D Montesi, MA Zamboni, in The International Conference on Computing Technology, Information Security and Risk Management (CTISRM2016). Social media investigations using shared photos, (Dubai, 2016), p. 47

19. M Moltisanti, A Paratore, S Battiato, L Saravo, in Image Analysis and Processing - ICIAP 2015 - 18th International Conference, Genoa, Italy, September 7-11, 2015, Proceedings, Part II. Image manipulation on facebook for forensics evidence (Springer, Genoa, 2015), pp. 506-517

20. Z Wang, AC Bovik, HR Sheikh, EP Simoncelli, Image quality assessment: from error visibility to structural similarity. IEEE Trans. Image Process. 13(4), 600-612 (2004)

21. D Bolton, youtube-dl documentation. github.com/rg3/youtube-dl/blob/ master/README.md\#readme. Accessed 22 Sept 2017

22. M Chen, J Fridrich, M Goljan, J Lukáš, Determining image origin and integrity using sensor noise. IEEE Trans. Inf. Forensic Secur. 3(1), 74-90 (2008)

23. M Goljan, J Fridrich, T Filler, in Publisher: Proc. SPIE 7254 IS\&T/SPIE Electronic Imaging. Large scale test of sensor fingerprint camera identification, (2009), pp. 72540-72540. International Society for Optics and Photonics

24. S Taspinar, M Mohanty, N Memon, in 2016 IEEE International Workshop on Information Forensics and Security (WIFS). Source camera attribution using stabilized video (IEEE, Abu Dhabi, 2016), pp. 1-6 
25. M Goljan, J Fridrich, Camera identification from scaled and cropped images. Secur. Forensic Steganography Watermarking Multimedia Contents X. 6819, 68190 (2008)

26. D Shullani, O Al Shaya, M Iuliani, M Fontani, A Piva, in Proceeding of 2017 Tyrrhenian International Workshop on Digital Communications, Communications in Computer and Information Science, vol. 766, September 18-20, 2017, Palermo. A Dataset for forensic analysis of videos in the wild (2017), pp. 84-94

\section{Submit your manuscript to a SpringerOpen ${ }^{\circ}$ journal and benefit from:}

- Convenient online submission

- Rigorous peer review

- Open access: articles freely available online

- High visibility within the field

- Retaining the copyright to your article

Submit your next manuscript at $\gg$ springeropen.com 